\title{
Can the promise of radionuclide cardiac innervation imaging be fulfilled?
}

\author{
Mark I. Travin, MD, MASNC ${ }^{a}$ \\ a Division of Nuclear Medicine, Department of Radiology, Montefiore Medical Center and the, \\ Albert Einstein College of Medicine, Bronx, NY
}

Received Oct 29, 2021; accepted Oct 31, 2021

doi: $10.1007 / \mathrm{s} 12350-021-02866-6$

\section{See related article, pp. 3179-3188}

In the current era, nuclear cardiology, despite its potential to uniquely assess underlying fundamental processes of cardiac pathophysiology, remains predominantly stress myocardial perfusion imaging (MPI) to assess coronary artery disease (CAD). Given that alternative modalities such as coronary computed tomographic (CT) angiography and cardiac magnetic resonance (CMR) imaging are comparably effective for diagnosis and guidance of CAD management, in order to continue to thrive and prosper, nuclear cardiology must better use its inherent strengths to image underlying biologic and molecular processes. Such methodology is especially crucial to help better deal with cardiac dysfunction and associated arrhythmias, conditions expected to increase dramatically in prevalence over the next several decades, and that will result in high cardiac morbidity, mortality, and costs. Encouraging in this regard is the relatively recent development and popularity of radionuclide imaging to diagnose and help manage two major causes of heart failure (HF) - transthyretin (TTR) amyloidosis and cardiac sarcoidosis, showing clear advantages over alternative imaging and invasive modalities. At the same time, when direct treatment for a specifically identified etiology of HF and arrhythmic complications are not possible or insufficient, one must address the pathophysiologic processes

Reprint requests: Mark I. Travin, MD, MASNC, Division of Nuclear Medicine, Department of Radiology, Montefiore Medical Center and the, Albert Einstein College of Medicine, 111 E. 210th Street, Bronx, NY 10467-2490; mtravin@attglobal.net

J Nucl Cardiol 2022;29:3189-93.

$1071-3581 / \$ 34.00$

Copyright (C) 2021 The Author(s) under exclusive licence to American Society of Nuclear Cardiology causing and worsening the situation. For sure current guideline-directed medical therapies (GDMT) and cardiac implantable electronic devices (CIED) that address these processes have yielded dramatic improvements in the lives of people with HF. Nevertheless, the approach to $\mathrm{HF}$ and associated arrhythmias needs to focus more directly on underlying fundamental mechanisms such as cellular dysfunction and destruction, the resultant inflammatory and fibrotic processes remodeling the myocardium, and related extracardiac systemic disruptions, all of which can potentially be assessed and tracked with radionuclide imaging. Unfortunately, investigatory imaging of deleterious cellular processes, such as ${ }^{99 m}$ Tc-annexin-V visualization of pathologic myocyte apoptosis, has not yet yielded clinical applications. ${ }^{1}$ There is exciting work seeking detailed imaging of cellular inflammatory and fibrotic responses to myocardial injury, but again no clinical application appears likely in the near future. ${ }^{2}$ Widespread recognition that the heart and cardiovascular system do not function in isolation, with recent exciting work showing that ${ }^{18} \mathrm{~F}$-FDG radionuclide visualization of organs such as the bone marrow and the amygdala in the brain reflects vascular inflammation and predicts adverse cardiac events, needs more investigatory work before these can be incorporated into routine practice. ${ }^{3}$

In contrast, there is already an approved wellstudied radionuclide tracer, iodine-123 metaiodobenzylguanidine, ( $\left.{ }^{123} \mathrm{I}-m \mathrm{IBG}\right)$, which can image a key component of an important physiologic entity interacting with the aforementioned $\mathrm{HF}$ pathophysiologic processes to undertake the body's response to cardiac injury and dysfunction, that is, the neurohormonal system. Neurohormonal processes consist of circulating hormones, such as epinephrine, norepinephrine (NE), arginine vasopressin, B-type natriuretic peptide (BNP), and mediators of the renin-angiotensin-aldosterone system (RAAS), as well as direct cardiac and vascular autonomic innervation by sympathetic and 
parasympathetic autonomic neurons. Together, these components serve to adjust cardiac output, blood volume, and vascular tone both to maintain sufficient circulation to body organs and tissues for normal activities, and to provide compensation for cardiac dysfunction. There has been investigational imaging of various hormonal components of the RAAS system, but no clinical utility is likely in the near future. Investigational radionuclide tracers have been used to image parasympathetic innervation and sympathetic post-synaptic receptors, but these too are not close to becoming clinically available.

Thus far, clinical success has been limited to imaging cardiac sympathetic (adrenergic) innervation with analogs of NE, including ${ }^{123} \mathrm{I}-m \mathrm{IBG}$ and the investigational positron emission tomographic (PET) tracer carbon-11 hydroxyephedrine $\left({ }^{11} \mathrm{C}\right.$-HED). First synthesized and imaged in human subjects about 40 years ago, ${ }^{123} \mathrm{I}-m \mathrm{IBG}$ imaging has consistently demonstrated unique clinical effectiveness in risk-stratifying patients with advanced heart failure with reduced ejection fraction (HFrEF) and, for over 10 years, has had an FDA-approved specific cardiac indication. Unfortunately, despite the well-recognized strength of cardiac ${ }^{123} \mathrm{I}-m$ IBG imaging, its promising clinical potential has not yet come to fruition. To consider how this might be overcome, it is worthwhile briefly reviewing what is established about its utility and strengths, its perceived short comings, and how to move forward, possibly with an analogous ${ }^{18} \mathrm{~F}$-based PET tracer as described in the investigation by Grkovski et al. ${ }^{4}$ in this issue of the journal.

\section{CONSISTENT EVIDENCE FOR THE UNIQUE RISK STRATIFICATION BENEFITS OF CARDIAC ADRENERGIC IMAGING}

Within a few years after the 1981 publication of the first human images of cardiac ${ }^{123} \mathrm{I}-m \mathrm{IBG}$ imaging, the tracer's ability to depict cardiac function differently from existing radionuclide cardiac tracers was well recognized. Initial work in the US, Japan and elsewhere, in humans and animals, explored potential utility of cardiac ${ }^{123}$ I- $m$ IBG imaging in a variety of cardiac conditions including ischemic heart disease especially in the setting of myocardial infarction, in hypertrophic cardiomyopathy and in dilated cardiomyopathy, including the tracer's ability to detect lethal arrhythmic risk. ${ }^{5}$ A key 1992 study by Merlet et al. demonstrated that in patients with $\mathrm{HFrEF}$, cardiac ${ }^{123} \mathrm{I}-\mathrm{mIBG}$ uptake as measured by the heart to mediastinum ratio (HMR) provided a wide differentiation in 25 -month mortality that was independent of and superior to conventional HF risk stratification variables including left ventricular ejection fraction (LVEF). ${ }^{6}$ Numerous small center studies led to combined multicenter data and literature metaanalyses reports that provided solid evidence of the independent risk stratification power of cardiac ${ }^{123} \mathrm{I}-$ $m$ IBG imaging in patients with HFrEF. ${ }^{7}$ These all culminated in the ADMIRE-HF (AdreView Myocardial Imaging for Risk Evaluation in Heart Failure) prospective multicenter international observation study of 961 patients with New York Heart Association (NYHA) Class II-III HF with LVEF $\leq 35 \%$. $^{8}$ The results of ADMIRE-HF were comprehensive and strong, with convincing 17-month risk stratification power, especially that patients having an HMR $\geq 1.6$ had extremely low hard event rates of $3 \%$ for cardiac death (versus $16.1 \%$ for HMR < 1.6 ) and $3.5 \%$ for potentially lethal ventricular arrhythmias (versus $10.4 \%$ for HMR $<1.6$ ). It is important to note that these arrhythmic events included sustained ( $>30$ seconds) ventricular tachycardia and ICD discharges rather than actual arrhythmic deaths. In a subanalysis of 201 patients with HMR $\geq$ 1.6, many of whom did not have an ICD during the study, there was only 1 arrhythmic death, meaning that cardiac ${ }^{123}$ I- $m$ IBG imaging can, within a cohort of highrisk HF patients, identify a subgroup for whom benefit of an ICD may be less than the potential risk of adverse device complications. The ADMIRE-HFX analysis that extended ADMIRE follow-up to 2 years, found that fatal and potentially fatal arrhythmic events were absent in subjects with an HMR of $\geq 1.8$, but in contrast occurred in $6-10 \%$ of subject with HMR less than 1.6 . $^{9}$

Another important finding from ADMIRE-HF was that cardiac ${ }^{123} \mathrm{I}-\mathrm{mIBG}$ imaging predicted adverse cardiac events independently of conventionally used HF risk stratification variables such as LVEF and BNP, with a subanalysis showing a $22.7 \%$ risk classification improvement over the Seattle Heart Failure Model combination of demographic, imaging, laboratory, and therapeutic parameters. ${ }^{10}{ }^{123}$ I- $m$ IBG imaging has also been shown to predict lethal arrhythmic events independently of electrocardiographic parameters. ${ }^{11}$

\section{BARRIERS TO USE OF ADRENERGIC IMAGING}

Based mostly on ADMIRE-HF results, in 2013, ${ }^{123}$ I- $m$ IBG (AdreView ${ }^{\mathrm{TM}}$ ) was approved for cardiac imaging by the Food and Drug Administration to "help identify patients with lower 1- and 2-year mortality risks, as indicated by an $[\mathrm{HMR}] \geq 1.6$," although utility was considered not established for "selecting a therapeutic intervention or for monitoring the response to therapy nor for using the [HMR] to identify a patient with a high risk for death" [https://www.accessdata.fd a.gov/drugsatfda_docs/label/2013/022290s001lbl.pdf]. Nevertheless, despite tracer approval and the wealth of 
robust high-quality data available, ${ }^{123} \mathrm{I}-m \mathrm{IBG}$ has not achieved significant cardiac clinical use in the US and has not been incorporated into major societal guidelines for assessment and management of HF and arrhythmias. A major obstacle has been the absence of a successfully performed prospective randomized trial demonstrating the benefit of ${ }^{123} \mathrm{I}-m \mathrm{IBG}$ imaging in directing ICD implantation, without which there is high fear of a potential fatal event if "unproven" ${ }^{123}$ I- $m$ IBG imaging is used to defer placing an ICD in a patient for whom societal guidelines advise otherwise. An attempted prospective study addressing this concern was stopped prematurely because of insufficient site and patient recruitment based in large part on these concerns. ${ }^{12}$

An additional disincentive has been high cost of the tracer in relation to reimbursement rates, with some private payers not providing any coverage for cardiac imaging. ${ }^{5}$ This situation has discouraged potential use of ${ }^{123}$ I- $m$ IBG imaging in clinical situations for which ICD implantation is uncertain that, if undertaken, could provide the practical experience and accumulation of data that would lead to enhanced understanding of the most suitable patients to image, give insight into effective use of image results, and provide guidance for more practically performable prospective investigations.

Another important barrier to ${ }^{123} \mathrm{I}-m \mathrm{IBG}$ use is discomfort with the limited information that can be derived from planar images. Many experts believe that a planar HMR provides insufficient detail to properly assess adrenergic innervation and reliably guide patient management, especially with regard to addressing arrhythmic risk. Single-photon emission-computed tomographic (SPECT) ${ }^{123} \mathrm{I}-m \mathrm{IBG}$ imaging provides more image detail, with visualization of electrically unstable regional innervation heterogeneity. With the addition of perfusion imaging, SPECT can show arrhythmogenic areas of denervation/perfusion mismatch.

SPECT ${ }^{123} \mathrm{I}-m$ IBG data studies suggest that adrenergic imaging needs to be approached differently from the customary perfusion imaging paradigm that more extensive and severe abnormalities predict a poorer prognosis. For adrenergic imaging, the regional pattern on SPECT imaging may be more important, with intermediate degrees of innervation abnormalities predicting increased arrhythmic risk. ${ }^{13,14}$ Unfortunately, the diagnostic quality of current SPECT ${ }^{123} \mathrm{I}-m \mathrm{IBG}$ images is often subpar, especially in patients with severe HF who have reduced to minimal tracer uptake resulting in poor resolution that, along with frequent overlapping extracardiac uptake, can make assessment and characterization of abnormalities difficult.

\section{MIGHT PET BE THE WAY TO GO?}

There is promising work showing that SPECT ${ }^{123} \mathrm{I}-$ $m$ IBG with a cadmium-zinc-telluride (CZT) camera, having high sensitivity and better energy resolution for simultaneous adrenergic and perfusion imaging, can provide clearer tissue detail to identify arrhythmic trigger zones. ${ }^{15}$ However, for a variety of reasons, PET imaging may be best. PET has numerous advantages including superior image quality with better spatial resolution, and the ability to perform dynamic imaging with accurate uptake and washout quantitation. In addition, in contrast to SPECT, with PET, there is more potential to create tracers with varying uptake properties that can provide pathophysiologic detail of differing adrenergic innervation abnormalities. ${ }^{16,17}$ PET cardiac adrenergic imaging has been studied for many years, mostly using ${ }^{11} \mathrm{C}-\mathrm{HED}$. The Prediction of Arrhythmic Events with Positron Emission Tomography (PAREPET) study of 204 ischemic cardiomyopathic subjects with $\mathrm{LVEF} \leq 35 \%$, undergoing ${ }^{11} \mathrm{C}$-HED adrenergic imaging found that the $\%$ innervation shown by ${ }^{11} \mathrm{C}$ HED imaging predicted 4-year occurrence of sudden cardiac arrest (SCA), while infarct volume, BNP, and LVEF did not. ${ }^{18}$ Furthermore, combining PET sympathetic denervation imaging with $\mathrm{LV}$ end diastolic volume, creatinine, and no angiotensin innervation was able to identify an extremely low risk subgroup (SCA < $1 \%$ /year) within this otherwise high-risk group.

While ${ }^{11} \mathrm{C}-\mathrm{HED}$ is considered an excellent sympathetic innervation tracer, the need for an onsite or nearby cyclotron makes it impractical for most clinicians. More clinically feasible ${ }^{18} \mathrm{~F}$ compounds are under active investigation, including ${ }^{18} \mathrm{~F}$-Flubrobenguane (FBBG, also known as ${ }^{18}$ F-LMI-1195) ${ }^{16}$ and the tracer F-18 meta-fluorobenzylguanidine $\left({ }^{18} \mathrm{~F}-\mathrm{MFBG}\right)$ investigated by Grkovski et al in this issue of the Journal of Nuclear Cardiology. ${ }^{4}$ Based on the group's first-in-human phaseI study of ${ }^{18} \mathrm{~F}-\mathrm{MFBG}$ in patients with neuroblastoma and pheochromocytoma/paragangliomas showing safety and favorable biodistribution and kinetics with good targeting of neuroendocrine malignancies, ${ }^{19}$ Grkovski et al performed concurrent dynamic and static ${ }^{18} \mathrm{~F}-\mathrm{MFBG}$ cardiac imaging in 16 of these patients with no known cardiac dysfunction, with ${ }^{123}$ I- $m$ IBG imaging also performed as part of standard of care. There was rapid and sustained myocardial ${ }^{18}$ F-MFBG tracer uptake (plateau at 30 seconds post-injection), and moderate correlation ( $\rho=0.67)$ of image HMRs with those of ${ }^{123}$ I- $m$ IBG SPECT (although actual values were not comparable as imaging was at different times after tracer administration). There was also a good correlation ( $\rho=0.84$ at 4 hours) between ${ }^{18} \mathrm{~F}-\mathrm{MFBG}$ HMR and tracer distribution volume $\left(\mathrm{V}_{\mathrm{T}}\right)$. As noted by the authors, the ability to 
obtain dynamic uptake data with PET ${ }^{18}$ F-MFBG allows derivation of the $\mathrm{V}_{\mathrm{T}}$ that provides a surrogate marker of the density of the NET-1 (norepinephrine transporter-1) protein on sympathetic pre-synaptic membranes, thereby providing more detailed information about the health of adrenergic innervation. In addition, $V_{T}$ is a metric that, unlike HMR or washout rate (a commonly used prognostic parameter for ${ }^{123} \mathrm{I}-\mathrm{mIBG}$ imaging), does not depend on the post-injection time of imaging. The authors also note that their ${ }^{18} \mathrm{~F}-\mathrm{MFBG} \mathrm{V}_{\mathrm{T}}$ values are similar to those reported for ${ }^{11} \mathrm{C}$-HED and ${ }^{18} \mathrm{~F}$-Fluorbenguan. With regard to radiotracer chemistry, the authors detected less radiolabeled metabolites with ${ }^{18} \mathrm{~F}$ MFBG than what has been reported with ${ }^{123} \mathrm{I}-m \mathrm{IBG}$, allowing a more reliable true uptake estimate of the former. Finally, with ${ }^{18} \mathrm{~F}-\mathrm{MFBG}$, there is no need to consider blocking thyroid uptake of iodine as is the case with ${ }^{123} \mathrm{I}-m \mathrm{IBG}$.

\section{MOVING FORWARD}

Both of the described ${ }^{18} \mathrm{~F}$ compounds currently under investigation can provide reinvigoration of the quest to realize the promising clinical potential of cardiac adrenergic imaging. As outlined in the section, skillful strategies will be needed for success. Among these are performance of efficient investigatory studies with high likelihood of success that will provide solid evidence of a reasonably cost-effective clinical outcome benefit that supersedes or significantly adds to other imaging techniques. Image interpretation will need to take advantage of the more detailed quantitative data obtained with PET, with more skillful consideration of the specific parameters assessed that is likely not simple cut-off thresholds but rather ranges of image measurement. Using artificial intelligence to combine these image data with clinical parameters can potentially produce individualized patient risk determination and unique management. It will be important to work in an interdisciplinary fashion with experts in other advanced imaging modalities, such as cardiac MRI and advanced echocardiography that can, together with radionuclide imaging, provide detailed tissue characterization, tissue tracking, pressure/flow characterization, and volumetric characterization, providing a comprehensive assessment of LV dysfunction and arrhythmogenicity. ${ }^{20}$ Finally, it will be crucial to work with other subspecialists, particularly heart failure and electrophysiology experts, to meet the needs of those on the front lines of direct patient therapeutics, demonstrated by work combining adrenergic imaging with cardiac MRI and electroanatomic mapping to improve the outcome of ventricular ablation procedures. $^{21}$ The success of radionuclide autonomic imaging will depend on its contribution to other technologies to improve the lives of people with cardiac disease.

\section{POTENTIAL STRATEGIES TO ENHANCE AND CONVINCINGLY DEMONSTRATE COST- EFFECTIVE CLINICAL UTILITY OF RADIONUCLIDE CARDIAC INNERVATION IMAGING}

- Formulate effective, efficient prospective clinical trials focusing on directing patient management that will improve clinical outcome

Perform studies investigating a variety of patient treatment strategies-medical management, cardiac implantable electronic devices, and therapeutic procedures such as arrhythmia ablation

$\bigcirc$ Broaden patient selection

Consider those in whom published guidelines do not provide clear direction

- Further explore innervation imaging of entities other than HF/arrhythmias, such as various nonischemic cardiomyopathies, cardiac dysfunction post-chemotherapy, complications that occur in transplanted hearts.

- If sufficient clinical use becomes economically feasible, registries can be created

- Enhance technical capabilities, with further investigation and development of:

Pre-synaptic adrenergic PET tracers, such as ${ }^{18} \mathrm{~F}$ MFBG and ${ }^{18} \mathrm{~F}-\mathrm{FBBG}$, which provide more accurate and detailed image characterization and quantitation, and well as other PET tracers depicting the various components of normal and abnormal pre-synaptic sympathetic function.

$\bigcirc$ Tracers that can image parasympathetic and postsynaptic sympathetic pathophysiology

Improved single-photon imaging capability, such as with cadmium-zinc-telluride cameras

$\bigcirc$ Multimodality imaging with technologies such cardiac magnetic resonance and electroanatomic mapping

- Improved image interpretive techniques

Determine the best image parameters to assess.

Cut-off thresholds versus ranges of abnormality

- Combine image results, clinical data, and findings from other imaging modalities using artificial intelligence with machine learning to direct individualized patient management 


\section{References}

1. Niu G, Chen X. Apoptosis imaging: Beyond Annexin V. J Nucl Med 2010;51:1659-62.

2. Bengel FM, Ross TL. Emerging imaging targets for infiltrative cardiomyopathy: Inflammation and fibrosis. J Nucl Cardiol 2019;26:208-16.

3. Osborne MT, Abohashem S, Zureigat H, Abbasi TA, Tawakol A. Multimodality molecular imaging: Gaining insights into the mechanisms linking chronic stress to cardiovascular disease. J Nucl Cardiol 2021;28:955-66.

4. Grkovski M, Zanzonico P, Humm J, Narula J, Pandit-Taskar N. F18 meta-fluorobenzylguanidine PET imaging of myocardial sympathetic innervation. J Nucl Cardiol 2021.

5. Travin MI, Matsunari I, Thomas GS, Nakajima K, Yoshinaga K. How do we establish cardiac sympathetic nervous system imaging with ${ }^{123}$ I-mIBG in clinical practice? Perspectives and lessons from Japan and the US. J Nucl Cardiol 2019;26:1434-51.

6. Merlet P, Valette H, Dubois-Randé J, Moyse D, Duboc D, Dove P, et al. Prognostic value of cardiac metaiodobenzylguanidine in patients with heart failure. J Nucl Med 1992;33:471-7.

7. Verberne HJ, Brewster LM, Somsen GA, van Eck-Smit BL. Prognostic value of myocardial 123I-metaiodobenzylguanidine (MIBG) parameters in patients with heart failure: A systematic review. Eur Heart J 2008;29:1147-59.

8. Jacobson AF, Senior R, Cerqueira MD, Wong ND, Thomas GS, Lopez VA, et al. Myocardial iodine-123 meta-iodobenzylguanidine imaging and cardiac events in heart failure. Results of the prospective ADMIRE-HF (AdreView Myocardial Imaging for Risk Evaluation in Heart Failure) study. J Am Coll Cardiol 2010;55:2212-21.

9. Narula J, Gerson M, Thomas GS, Cerqueira MD, Jacobson AF. 123I-MIBG imaging for prediction of mortality and potentially fatal events in heart failure: The ADMIRE-HFX Study. J Nucl Med 2015;56:1011-8.

10. Ketchum E, Jacobson A, Caldwell J, Senior R, Cerqueira M, Thomas G, et al. Selective improvement in Seattle heart failure model risk stratification using iodine-123 meta-iodobenzylguanidine imaging. J Nucl Cardiol 2012;19:1007-16.

11. Tamaki S, Yamada T, Okuyama Y, Morita T, Sanada S, Tsukamoto Y. Cardiac iodine-123 Metaiodobenzylguanidine imaging predicts sudden cardiac death independently of left ventricular ejection fraction in patients with chronic heart failure and left ventricular systolic dysfunction: Results from a comparative study with signal-averaged electrocardiogram, heart rate variability, and QT dispersion. J Am Coll Cardiol 2009;53:426-35.

12. ADMIRE-ICD: International Study to Determine if AdreView Heart Function Scan Can be Used to Identify Patients With Mild or Moderate Heart Failure (HF) That Benefit From Implanted Medical Device, ADMIRE-ICD, at Clin trials: https://www.clinica 1trials.gov/ct2/show/NCT02656329, accessed 10/29/21.

13. Travin MI, Henzlova MJ, van Eck-Smit BLF, Jain J, Carrió I, Folks RD, et al. Assessment of ${ }^{123} \mathrm{I}-\mathrm{mIBG}$ and ${ }^{99 \mathrm{~m}} \mathrm{Tc}$-tetrofosmin single-photon emission computed tomographic images for the prediction of arrhythmic events in patients with ischemic heart failure. J Nucl Cardiol 2017;24:377-91.

14. Verschure DO, Poel E, Travin MI, Henzlova MJ, Jain D, Jacobson $\mathrm{AF}$, et al. A simplified wall based model for regional innervation/ perfusion mismatch assessed by cardiac ${ }^{123} \mathrm{I}-\mathrm{mIBG}$ and rest ${ }^{99 \mathrm{~m}} \mathrm{Tc}$ tetrofosmin SPECT to predict arrhythmic events in ischemic heart failure. Eur Heart J Cardiovasc Imaging 2021. https://doi.org/10. 1093/ehjci/jeab132.

15. D'estanque E, Hedon C, Lattuca B, et al. Optimization of a simultaneous dual-isotope ${ }^{201} \mathrm{Tl} /{ }^{123} \mathrm{I}$-MIBG myocardial SPECT imaging protocol with a CZT camera for trigger zone assessment after myocardial infarction for routine clinical settings: Are delayed acquisition and scatter correction necessary? J Nucl Cardiol 2017;24:1361-9.

16. Zelt JGE, Britt D, Mair BA, Rotstein BH, Quigley S, Walter O, et al. Regional distribution of fluorine-18-flubrobenguane and carbon-11-hydroxyephedrine for cardiac PET imaging of sympathetic innervation. J Am Coll Cardiol Img 2021;14:1425-36.

17. Lautamäki R, Sasano $T$, Higuchi $T$, et al. Multiparametric molecular imaging provides mechanistic insights into sympathetic innervation impairment in the viable infarct border zone. J Nucl Med 2015;56:457-63.

18. Fallavollita JA, Heavey BM, Luisi AJ Jr, Michalek SM, Baldwa S, Mashtare TL, et al. Regional myocardial sympathetic denervation predicts the risk of sudden cardiac arrest in ischemic cardiomyopathy. J Am Coll Cardiol 2014;63:141-9.

19. Pandit-Taskar N, Zanzonico P, Staton KD, Carrasquillo JA, ReidyLagunes D, Lyashchenko S, et al. Biodistribution and dosimetry of ${ }^{18}$ F-meta-fluorobenzylguanidine: A first-in-human PET/CT imaging study of patients with neuroendocrine malignancies. J Nucl Med 2018;59:147-53.

20. Argulian E, Narula J. Advanced cardiovascular imaging in heart failure. J Am Coll Cardiol HF 2021;9:699-709.

21. Imanli H, Ume KL, Jeudy J, Bob-Manuel T, Smith MF, Chen W, et al. Ventricular tachycardia (VT) substrate characteristics: insights from multimodality structural and functional imaging of the VT substrate using cardiac MRI scar, ${ }^{123}$ I-metaiodobenzylguanidine SPECT innervation, and bipolar voltage. J Nucl Med 2019;60:79-85.

Publisher's Note Springer Nature remains neutral with regard to jurisdictional claims in published maps and institutional affiliations. 